\title{
Robert Lessmann
}

\section{Drogenökonomie und internationale Politik}

Die Auswirkungen der Antidrogen-Politik der USA auf Bolivien und Kolumbien

Vervuert Verlag, Frankfurt am Main, 199, 305 S., DM 56,--

(Schriftenreihe des Instituts für Iberoamerika-Kunde Hamburg, Bd. 41)

Mit der partiellen Ausnahme Mexikos blieb der Stellenwert Lateinamerikas in der USAußenpolitik trotz der geographischen Nähe verhältnismäßig gering, solange dort keine Entwicklungen eintraten, die Washington als Gefährdung der eigenen (Sicherheits-) Interessen perzipierte. So wurde die Agenda der US-Lateinamerikapolitik bis zum Ende der 80er Jahre vom Zentralamerika-Konflikt dominiert, während die Beziehungen zu Südamerika wenig Aufmerksamkeit fanden. Seit der Befriedung der Isthmusregion und dem Ende des Kalten Krieges bewertet Washington den Drogenhandel als größtes Einzelproblem der interamerikanischen Beziehungen. Angesichts des Siegeszugs von Kokain und Crack auf dem illegalen Drogenmarkt der Vereinigten Staaten haben sich die US-Regierungen bemüht, die politischen Autoritäten der Herkunftsländer des Suchtstoffs für ein verstärktes Engagement gegen den Drogenhandel zu gewinnen. In seiner Publikation, die die gekürzte Fassung einer an der Universität Wien vorgelegten Dissertation darstellt, thematisiert Lessmann die Auswirkungen sowohl der Koka- und Kokainproduktion als auch der maßgeblich von den USA beeinflußten drogenpolitischen Gegenmaßnahmen auf Wirtschaft, Gesellschaft und Politik Boliviens und Kolumbiens.

Nach der Präsentation einiger Basisinformationen zum Drogenrohstoff Koka und der Beschreibung der Charakteristika des Suchtstoffs Kokain stellt der Autor in Kapitel 3 die Grundzüge der Drogenproblematik und -politik in den USA dar. Er verweist auf die Neigung der US-Regierungen, zumindest einen bedeutenden Teil der Verantwortung für das heimische Drogenproblem den jeweiligen Herkunftsländern der Suchtstoffe anzulasten und legt dar, wie Washington angesichts des Scheiterns der internen Verbots- und Repressionspolitik die Maßnahmen zur Unterbindung der illegalen Kokaineinfuhren sukzessive verstärkt und dabei unter dem Motto going to the source auch den Druck auf die Regierungen der Produzentenländer spürbar erhöht hat. Mit der 1986 eingeführten certification wurde die gesamte bilaterale Zusammenarbeit drogenpolitischen Gesichtspunkten unterworfen: Seither haben Länder, denen das Weiße Haus eine unzureichende Kooperation bei der Bekämpfung des Drogenhandels attestiert, nicht nur den Wegfall der US-Wirtschaftshilfe, sondern auch ein Veto Washingtons gegen die Kreditvergabe von Weltbank, IWF und interamerikanischer Entwicklungsbank zu gewärtigen. Die jährliche certification erwies sich vor allem im Falle des ökonomisch schwachen und massiv von internationaler Entwicklungshilfe abhängigen Bolivien als effizientes Instrument, um den drogenpolitischen Forderungen und Erwartungen der USA Nachdruck zu verleihen. Seit Mitte der 80er Jahre sehen sich die bolivianischen Regierungen mit dem Dilemma konfrontiert, daß sie den drogenpolitischen Vorgaben der USA nur zum Teil entsprechen können, um den Widerstand der gut organisierten Kokabauern zu begrenzen, die eine Einstellung des Kokaanbaus 
von angemessenen Kompensationsleistungen abhängig machen, für die La Paz jedoch die finanziellen Mittel fehlen. Insbesondere die von Washington geforderte Einbeziehung des Militärs in die Programme zur Bekämpfung des Drogenhandels haben langanhaltende innenpolitische Kontroversen ausgelöst. Auch Kolumbien war unter den gegebenen innenpolitischen Bedingungen nur partiell bzw. zeitweise bereit oder in der Lage, der drogenpolitischen Linie Washingtons zu folgen, wobei die Forderung nach einer Auslieferung von Drogenhändlern an die US-Justiz bald zum zentralen Streitpunkt im Verhältnis der beiden Staaten avancierte. Lessmann hebt hervor, daß die USA mit ihrem in die Andenstaaten getragenen war on drugs tatsächlich in erster Linie drogenpolitische Zwecke verfolgen und nicht, wie of tmals vermutet oder behauptet, die Drogenproblematik nur als Vorwand benutzen, um geostrategisch-imperialistische Ziele zu realisieren. Das drogenpolitische Engagement in den sog. source countries erfüllt dabei auch immer innenpolitische Funktionen, wobei symbolische Aspekte überwiegen.

Trotz der Vermittlung zahlreicher wichtiger Einzelinformationen und der Darlegung einiger zentraler Wirkungszusammenhänge vermag die Studie aufgrund einer Reihe fundamentaler Mängel und Defizite insgesamt nicht zu überzeugen. Eine der wesentlichen Schwächen beruht auf der vollkommen veralteten Datenbasis. Nun hinkt jede Untersuchung, die sich einem aktuellen, ständig im Fluß befindlichen Thema wie dem Drogenhandel widmet, zum Zeitpunkt ihres Erscheinens zwangsläufig den neuesten Entwicklungen und Trends hinterher; es ist aber nicht zu akzeptieren, wenn die Argumentation einer 1996 veröffentlichten Studie sich auf Daten stützt, die im wesentlichen die Situation der Jahre 1988-1990 dokumentieren. Da dem Autor somit die empirische Basis fehlt, um die konkreten Implikationen und Folgen der 1989 von der Bush-Administration lancierten Andeninitiative erfassen zu können, vermag er dem Leser nicht mehr als Mutmaßungen zu offerieren. So ist es schlicht unredlich, den Fehlschlag der bolivianischen Projekte alternativer Entwicklung zu konstatieren, ohne sich darum zu kümmern, was sich auf diesem Gebiet nach 1990 getan hat.

Aber auch das veraltete Zahlenmaterial wird in einer wenig sorgfältigen Weise verwendet: So werden zumindest drei verschiedene (und extrem voneinander abweichende) Angaben zur Menge der bolivianischen Kokabauern präsentiert; ebenso uneinheitlich sind die demographischen Daten zur Kokaanbauzone Chapare. Zudem sind die Berechnungen zu den Einkommen der Kokafarmer schlichtweg falsch.

Eine weitere Schwäche der Arbeit besteht in den zahlreichen und ermüdenden Wiederholungen. Die Zusammenfassungen am Ende der Kapitel reduzieren sich auf reine Repetitionen des zuvor Gesagten ohne jeglichen analytischen Zugewinn. Unter Berücksichtigung des "Resümees" in Kap. 8 tauchen mehrere Gedankengänge (z.T. in kaum modifizierten Versionen) dreimal in der Studie auf. Bei Verzicht auf diese Dopplungen und Redundanzen hätte der Umfang des Buches wohl um ein Viertel reduziert werden können. Gleichzeitig werden bestimmte methodische und analytische Schwächen in der Darstellung durch die Wiederholung besonders deutlich.

Der Autor agiert wenig souverän und seriös, wenn er aus einigen wenigen fragwürdigen Indizien, darunter v.a. Äußerungen von drittrangigen Vertretern der US-Regierung, Pläne 
für eine umfassende und allein von den Interessen der USA bestimmten Militarisierung des war on drugs in Lateinamerika ableitet. Der in diesem Zusammenhang von Lessmann mehrfach erwähnte "Thurman-Plan" scheint der Phantasie des Autors näher zu stehen als der politischen Realität. Er nimmt dabei offensichtlich einige Statements zum Nennwert, die in Wirklichkeit wohl primär taktischen Charakter besaßen. Weil diese tendentiell verschwörungstheoretischen Szenarien in der Folgezeit nicht durch faktische Entwicklungen erhärtet wurden, hätte der Autor gut daran getan, die entsprechenden Thesen vor der Drucklegung des Buches aus dem Text zu tilgen.

In den Darlegungen ist of der rote Faden nicht zu erkennen, die Aussagen sind bisweilen widersprüchlich bzw. nicht recht nachvollziehbar. Was soll etwa der mehrmals hervorgehobene Befund einer "rücksichtslosen Ausbeutung" und eines "Faustrechts des Stärkeren in einer Situation eines freien (!) Konkurrenzkapitalismus", wenn die Tagelöhner, auf die diese Charakterisierung abzielt, in der Kokawirtschaft ungleich mehr verdienen als in allen anderen Wirtschaftszweigen. Eine Aussage wie: "Der Boom im Chapare ist auf fragilen Fundamenten einer ökonomischen Monokultur und eines externen Absatzmarktes aufgebaut, der zudem noch illegal ist" enthält gleich drei Ungereimtheiten: Weder betreibt die Mehrzahl der Kokabauern eine reine Monokultur, noch ist der Exportmarkt für Kokain fragiler als der für legale Produkte, und drittens ist die Illegalität konstituierendes Element des Booms. Die gravierende Wirtschaftskrise, die die Situation Boliviens in den 80er Jahren maßgeblich geprägt hat, gerät kaum ins Blickfeld des Verfassers; außerdem übernimmt er die dutch disease-Diagnose für die bolivianische Koka(in)ökonomie in einer sehr unkritischen Weise.

Gegenüber dem Bolivien-Kapitel fallen die Ausführungen zu Kolumbien qualitativ noch weiter ab. Dabei wird dem eigentlichen Thema der Untersuchung nur wenig Raum gegeben, was der Autor damit begründet, daß die schlechte Datenlage eine einigermaßen präzise Erfassung des Einflusses der USA auf die kolumbianische Drogenpolitik nicht zulasse. Dem wäre entgegenzuhalten, daß Lessmann seine Materialbasis nicht unwesentlich hätte bereichern können, wenn er die einschlägige US-amerikanische Fachliteratur zur Kenntnis genommen hätte; die im Literaturverzeichnis aufgeführten Artikel von Bruce Bagley sind im besten Fall wohl nur sehr oberflächlich rezipiert worden. Überhaupt werden die Beiträge von Forschern aus den USA in der gesamten Studie weitgehend ignoriert, während intensiver Gebrauch von offiziellen Dokumenten (Kongreßhearings u.a.) gemacht wird. Andererseits lehnt der Autor bei vielen Aspekten der Thematik - insbesondere im Kolumbien gewidmeten Teil der Arbeit - seine Darlegungen allzu eng an einige wenige Veröffentlichungen an, deren Argumentationsführung er oft über mehrere Seiten folgt. Angesichts eines solchen Vorgehens ist der Ertrag der immer wieder hervorgehobenen Feldforschung nicht zu erkennen. Vor einem Exkurs über die sicarios von Medellín weist Lessmann darauf hin, daß er seine Quellen zum Zwecke des Schutzes der Informanten nicht offenlegen werde, um in der Folge eher allgemeine Aussagen zu präsentieren, wie sie jeder gute Zeitungsartikel zu diesem Thema enthält. 
Obwohl der Text auch eine Reihe von argumentativen Lichtblicken enthält, reichen diese nicht aus, um den wenig soliden Gesamteindruck der Monographie zu korrigieren: Insgesamt wird die Untersuchung der Komplexität des behandelten Themas nicht gerecht; weder eignet sie sich als Einstieg in die Problematik, noch vermag sie einem Kenner der Materie nennenswerte zusätzliche Einblicke zu vermitteln.

Karl-Dieter Hoffmann

\section{Otto Luchterhandt (Hrsg.)}

Neue Regierungssysteme in Osteuropa und der GUS: Probleme der Ausbildung stabiler Machtinstitutionen

Berlin Verlag Arno Spitz, Berlin, 1996, DM 78,--

(Osteuropaforschung, Schriftenreihe der Deutschen Gesellschaft für Osteuropa, Bd. 37)

Mit dem Kollaps des Systemmodells kommunistischer Einparteienstaat in Osteuropa ist die nach dem Auseinanderbrechen der formalen Bundesstaaten Sowjetunion, Jugoslawien und Tschechoslowakei stark angewachsene Staatenfamilie im ehemaligen geopolitischen Einflußbereich der UdSSR dem westlichen Modell des demokratischen Verfassungsstaates nähergerückt. Die Probleme des gleichzeitigen Ablösungs- und Annäherungsprozesses der alten und neuen Staaten spiegeln sich in den Herausforderungen, die an die Beobachter und Kommentatoren des Wandels gestellt sind: Gefragt ist nicht mehr nur auf Sprach- und Landeskunde gestütztes Insiderwissen, sondern auch theoretische Tiefe in der Systemvergleichung.

Das Buch, das die Beiträge einer Tagung der Fachgruppe Politikwissenschaft der Deutschen Gesellschaft für Osteuropakunde vom November 1994 zusammenfaßt, versucht eine Bestandsaufnahme, wie die Staaten Osteuropas und der früheren Sowjetunion die Transformation ihrer politischen Systeme bewältigt haben. Der Untertitel verschleiert zum Teil das Problem: Stabile Machtstrukturen hat es vor der weitgehend überraschenden Wende in Osteuropa zweifelsohne gegeben; zu fragen wäre, wie weit die Herausbildung demokratischer Strukturen bislang gelungen ist.

Aufgrund der offensichtlichen Präferenz in Osteuropa, dem Staatspräsidenten eine starke Rolle zuzubilligen, handelt es sich eigentlich um ein Buch über Präsidentialismus, denn fünf der insgesamt sieben Beiträge widmen sich ausschließlich oder wesentlich diesem Phänomen. Winfried Steffani legt in einem theoretischen Beitrag (Parlamentarisch-präsidentielle "Mischsysteme"? Bemerkungen zum Stand der Forschung in der Politikwissenschaft) seine Auffassung einer Zwei-Formen-Lehre dar, in deren Mittelpunkt er die Möglichkeit der Abberufung der Regierung durch das Parlament aus politischen Gründen als primäres Unterscheidungskriterium von parlamentarischen und präsidentiellen Regierungssystemen stellt und konsequenterweise die Einordnung als präsidentiell-parlamentarische 